\title{
STUDIES ON VUV LASING WITH NEON-LIKE ALUMINUM IONS
}

\author{
B. RahMANI* \\ Tokyo Institute of Technology \\ 2-12-1 O-Okayama, Meguro-ku, Tokyo 152, Japan \\ (Received March 18, 1997; revised version July 10, 1997; \\ in final form February 12, 1998)
}

The collisional-radiative equilibrium model has been developed in order to investigate the population inversion on aluminum plasma. The population inversion has been established between lower $3 s\left({ }^{3} P_{1}^{0},{ }^{1} P_{1}^{0}\right)$ and upper $3 p\left({ }^{3} P_{0},{ }^{1} S_{0}\right)$ levels. The vacuum ultra-violet lasing on neon-like aluminum ions provides wavelengths of $111.9 \mathrm{~nm}, 143.2 \mathrm{~nm}, 157.3 \mathrm{~nm}$. The rate of relative density population, between lower $3 s^{1} P_{1}^{0}$ and upper $3 p{ }^{3} P_{0}$ levels, reaches its highest value of 7.16 at the electron density of $10^{19} \mathrm{~cm}^{-3}$. The modified expression of optical escape factor, in the case of Doppler profile, was introduced in the calculation of opacity effect on the gain magnitudes. The resonance radiation trapping by $3 d$ configuration alters drastically the gain values. A gain greater than $1 \mathrm{~cm}^{-1}$ was found between lasing levels $3 s^{3} P_{1}^{0}$ and $3 p^{3} P_{0}$. The enhancement of gain was due to the pumping of the upper level by dielectronic recombination process from the ground state of fluorine-like and by the inner-shell ionization process from excited levels of sodium-like ions.

PACS numbers: 42.55.Lt, $52.20 . \mathrm{Hv}$

\section{Introduction}

The electron pumping of Ne-like system was considered as a source for vacuum ultra-violet (VUV) lasing [1-9]. This is connected with the fact that the rates for electron collisional excitation from the $1 s^{2} 2 s^{2} 2 p^{6}$ ground state to the $1 s^{2} 2 s^{2} 2 p^{5} 3 p$ higher lasing levels are greater than the rates for excitation from the ground state to the $1 s^{2} 2 s^{2} 2 p^{5} 3 s$ lower lasing levels. The $3 s$ levels decay to the ground state by a dipole-allowed transition (fast decay), while the $3 p$ levels decay is due to the quadruple transition (slow decay).

In the quasi stationary plasma, the population inversion between the $3 p$ and $3 s$ levels of Ne-like ions has been investigated [1,4]. However, only 27 levels

${ }^{*}$ Permanent address: Institute of Electronics, University of Sciences and Technology of Oran, Bir-Eldjeer, M'naouer, B.P. 1505 Oran, Algeria. 
belonging to the configurations $1 s^{2} 2 s^{2} 2 p^{6}, 1 s^{2} 2 s^{2} 2 p^{5} 3 s, 1 s^{2} 2 s^{2} 2 p^{5} 3 p$, and $1 s^{2} 2 s^{2} 2 p^{5} 3 d$ of these ions have been taken into account. The ionization and dielectronic recombination processes, neglected in this simulation $[1,4]$, have shown a significant effect on the gain magnitudes $[2,10,11]$.

We present a study on the VUV lasing of Ne-like aluminum ions based on the collisional-radiative equilibrium (CRE) model which takes into account more excited levels up to the hydrogenic level $n=9$ for lasing ions. Both atomic processes, the ionization and dielectronic recombination, have been included in this model. The atomic structure of lasing ions consists of the $n l j$ and $n l$ coupling for $n=3$ and $n$ up to 5 , respectively, where $n$ is the principal quantum number. The recently published atomic data for $n$ up to 5 have been considered. The CRE model includes the sodium-like (Na-like) and fluorine-like (F-like) Al ions which are represented by their excited levels up to $n=7$ in $n l$ coupling and by the ground state for $\mathrm{Na-like}$ and $\mathrm{F}$-like $\mathrm{Al}$ ions, respectively.

\section{Atomic data and lasing structure}

The energy levels of $\mathrm{Na}$-like and $\mathrm{Ne}$-like $\mathrm{Al}$ ions were taken from Kaufman et al. [12]. The transition probability coefficients of Na-like were taken from Lindgard et al. [13] and those for Ne-like were taken from Hibbert et al. [14] and Loginov et al. [15]. The oscillator strengths for hydrogenic levels of Ne-like structure were well reproduced by using the fitting coefficients of Johnson [16]. The transition probability coefficients [14], calculated by the multiconfigurational optimised potential model, show good agreement with theoretical [17, 18] and experimental [19] values.

The energy levels of Ne-like Al ions shown in Fig. 1 play a major role in determining the overpopulation between specified sublevels for $n=3$. Besides, the

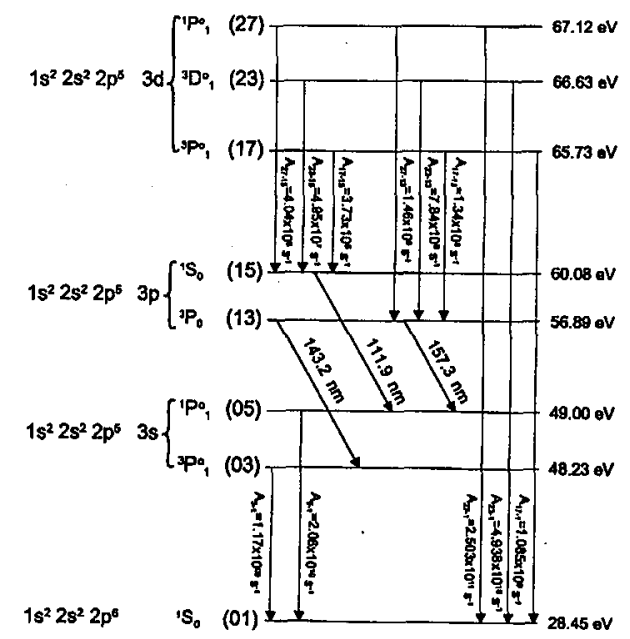

Fig. 1. A schematic diagram of some energy levels of neon-like aluminum. Ionization limit $-119.99 \mathrm{eV} . A_{13-5}=2.967 \times 10^{7} \mathrm{~s}^{-1}, A_{13-3}=8.439 \times 10^{8} \mathrm{~s}^{-1}, A_{15-5}=$ $1.934 \times 10^{9} \mathrm{~s}^{-1}$. 


\section{TABLE}

Lifetimes of $3 s$ and $3 p$ configurations of neon-like aluminum ions.

\begin{tabular}{c|lcc|l}
\hline \hline Numbers & \multicolumn{3}{|c|}{ States } & \multicolumn{1}{|c}{$\begin{array}{c}\text { Calculated } \\
\text { lifetimes [ns] }\end{array}$} \\
\hline 1 & $1 s^{2} 2 s^{2} 2 p^{6}$ & ${ }^{1} S_{0}$ & \\
2 & $1 s^{2} 2 s^{2} 2 p^{5} 3 s$ & ${ }^{3} P_{2}^{0}$ & \\
3 & $1 s^{2} 2 s^{2} 2 p^{5} 3 s$ & ${ }^{3} P_{1}^{0}$ & $6.390 \times 10^{-1}$ \\
4 & $1 s^{2} 2 s^{2} 2 p^{5} 3 s$ & ${ }^{3} P_{0}^{0}$ & \\
5 & $1 s^{2} 2 s^{2} 2 p^{5} 3 s$ & ${ }^{1} P_{1}^{0}$ & $4.852 \times 10^{-2}$ \\
6 & $1 s^{2} 2 s^{2} 2 p^{5} 3 p$ & ${ }^{3} S_{1}$ & 2.429 \\
7 & $1 s^{2} 2 s^{2} 2 p^{5} 3 p$ & ${ }^{3} D_{3}$ & 1.441 \\
8 & $1 s^{2} 2 s^{2} 2 p^{5} 3 p$ & ${ }^{3} D_{2}$ & 1.467 \\
9 & $1 s^{2} 2 s^{2} 2 p^{5} 3 p$ & ${ }^{3} D_{1}$ & 1.453 \\
10 & $1 s^{2} 2 s^{2} 2 p^{5} 3 p$ & ${ }^{1} D_{2}$ & 1.378 \\
11 & $1 s^{2} 2 s^{2} 2 p^{5} 3 p$ & ${ }^{1} P_{1}$ & 1.445 \\
12 & $1 s^{2} 2 s^{2} 2 p^{5} 3 p$ & ${ }^{3} P_{2}$ & 1.283 \\
13 & $1 s^{2} 2 s^{2} 2 p^{5} 3 p$ & ${ }^{3} P_{0}$ & 1.145 \\
14 & $1 s^{2} 2 s^{2} 2 p^{5} 3 p$ & ${ }^{3} P_{1}$ & 1.230 \\
15 & $1 s^{2} 2 s^{2} 2 p^{5} 3 p$ & ${ }^{1} S_{0}$ & $4.878 \times 10^{-1}$
\end{tabular}

collisional condition, the lifetime of the upper level must be greater than the lower level which is calculated with Eq. (1) with the use of the transition probability coefficients given by Hibbert et al. [14] and Loginov et al. [15]

$$
\tau_{i}^{-1}=\sum_{j<i} A_{i j} .
$$

The calculated values of lifetime by Eq. (1) for $3 s$ and $3 p$ configurations of Ne-like $\mathrm{Al}$ ions are given in Table. These values show that the radiative condition for population inversion between the lower levels of $3 s(3,5)$ and upper leyels of $3 p(13,15)$ configurations is fulfilled.

\section{Atomic processes and CRE scheme}

The population inversion was predicted in optically thin Ne-like system $[1,4]$ with only 27 levels of $n=3$ and without including the ionization and dielectronic recombination atomic processes. The elaborated models which have been recently developed for Ne-like system $[2,5,7,8]$ take into account more excited levels of lasing ions and include some levels of both $\mathrm{F}$-like and $\mathrm{Na}$-like ions. The atomic structure for Ne-like system includes excited levels up to $n=3$ in the model of Davis et al. [8], up to $n=6$ in the model of Apruzese el al. [5], up to $n=4$ in the model of Dasgupta et al. [2] and Holden et al. [7]. 
We have developed a CRE model taking into account more excited levels for Ne-like (Al IV) ions, including other aluminum ions and opacity effect. The atomic structure of lasing ions (Al IV) consists of ground state, levels in $n l j$ coupling for $n=3$, levels in $n l$ coupling for $n=4$ and 5 , and hydrogenic levels up to $n=9$. The Na-like (Al III) ions were represented by excited levels up to $n=7$ in $n l$ coupling while the aluminum atoms ( $\mathrm{Al} \mathrm{I}$ ), magnesium-like (AI II) and F-like (Al V) ions were supposed in their ground state

(1) $\mathrm{Al} \mathrm{III}(p)+\mathrm{e} \rightleftharpoons \mathrm{Al} \mathrm{III}(q)+\mathrm{e}$

(2) $\mathrm{Al} \mathrm{III}(p)+\mathrm{e} \longrightarrow \mathrm{Al} \mathrm{IV}(1)+\mathrm{e}+\mathrm{e}$

(3) Al III $(p)+\mathrm{e} \longleftarrow \mathrm{Al} \mathrm{IV}(1)+\mathrm{e}+\mathrm{e}$

(4) $\mathrm{Al} \mathrm{III}(p) \longrightarrow \mathrm{Al}$ III $(q)+h \nu$

(5) $\mathrm{Al} \mathrm{IV} \mathrm{(1)}+\mathrm{e} \longrightarrow \operatorname{Al}$ III $(p)+h \nu$

(6) $\mathrm{Al} \mathrm{IV}(i)+\mathrm{e} \rightleftharpoons \mathrm{Al} \mathrm{IV}(j)+\mathrm{e}$

(7) $\mathrm{Al} \mathrm{IV}(i)+\mathrm{e} \longleftarrow \operatorname{Al~V}(1)+e+e$

(8) $\mathrm{Al} \mathrm{IV}(i)+\mathrm{e} \longrightarrow \mathrm{Al} \mathrm{V}(1)+\mathrm{e}+\mathrm{e}$

(9) $\mathrm{Al} \mathrm{IV}(i) \longrightarrow \mathrm{Al} \mathrm{IV}(j)+h \nu$

(10) $\mathrm{Al} \mathrm{V} \mathrm{(1)}+\mathrm{e} \longrightarrow \mathrm{Al} \mathrm{IV}(i)+h \nu$

$(11) \mathrm{Al} \mathrm{IV}(1)+\mathrm{e} \longrightarrow \mathrm{Al} \mathrm{III*}(p) \longrightarrow \mathrm{Al}$ III $(p)+h \nu$

(12) $\mathrm{Al} \mathrm{V}(1)+\mathrm{e} \longrightarrow \mathrm{Al} \mathrm{IV}^{*}(i) \longrightarrow \mathrm{Al} \mathrm{IV}(i)+h \nu$.

The levels of Al III are designed by $p$ and $q$, while those of Al IV by $i$ and $j$. The coefficients of electron excitation rates are designed by $X(p, q)$ and $X(i, j)$ for $\mathrm{Na-like}(1)$ and Ne-like (6) ions, respectively. The ionization rates coefficients are designed by $S(p, 1)$ and $S(i, 1)$ for the ionization processes $(2)$ and $(8)$, respectively. The processes (3) and (7) are three-body recombination for both Na-like and Ne-like ions. The radiative recombination for both ions Al III (5) and Al IV (10) is designed by $\beta(p)$ and $\beta(i)$, respectively. The dielectronic recombination process is described by $\gamma(p)$ and $\gamma(i)$ for Al III (11) and Al IV (12), respectively. The spontaneous transition probabilities $A(p, q)(4)$ and $A(i, j)(9)$ for Na-like and Ne-like ions are replaced by $A(p, q) \Lambda(p q)$ and $A(i, j) \Lambda(i j)$ when the opacity effect is included in this model, where $\Lambda$ is the optical escape factor.

The population density of aluminum ions levels is obtained by solving the following system formed by 77 linear equations:

- equation for $\mathrm{Al}$ III levels

$$
\begin{aligned}
\frac{\mathrm{d} n(p)}{\mathrm{d} t} & =-n(p)\left[N_{\mathrm{e}} S(p, 1)+N_{\mathrm{e}} \sum_{p \neq q}^{19} X(p, q)+\sum_{p>q} A(p, q)\right]+\sum_{p \neq q}^{19} n(q) X(p, q) N_{\mathrm{e}} \\
& +\sum_{q>p} n(q) A(p, q)+N_{\mathrm{e}}\left[N_{\mathrm{e}} \alpha(p, 1)+\beta(p)+\gamma(p)\right] n(i)_{i=1},
\end{aligned}
$$


- equation for Al IV levels

$$
\begin{aligned}
\frac{\mathrm{d} n(i)}{\mathrm{d} t} & =-n(i)\left[N_{\mathrm{e}} S(i, 1)+N_{\mathrm{e}} \sum_{i \neq j}^{55} X(i, j)+\sum_{i>j} A(i, j)\right]+\sum_{i \neq j}^{55} n(j) X(i, j) N_{\mathrm{e}} \\
& +\sum_{j>i} n(j) N_{\mathrm{e}} A(i, j)+N_{\mathrm{e}}\left[N_{\mathrm{e}} \alpha(i, 1)+\beta(i)+\gamma(i)\right] n(l)_{l=1},
\end{aligned}
$$

where $n(i)_{i=1}, n(l)_{l=1}$ are the population density of ground state of Ne-like and F-like $\mathrm{Al}$ ions.

The change of atomic processes is very fast relative to the changing of plasma parameters. Therefore, the time dependence in the left side of Eqs. (2) and (3) is canceled. This linear system of equation was coupled to the neutrality equation.

\section{Rate coefficient}

The rate of excitation and of ionization has been calculated under the assumptions of Maxwellian distribution for electron velocity. In the literature, for each atomic process, there is no universal rate coefficient $[16,20-25]$. The main difference is due to the theoretical method used for functions of cross section. In this model we have used the following rate coefficients.

\subsection{Collisional excitation}

The collisional excitation rates $X(m, n)$ of optically allowed transitions are obtained by using the following equation $[24,26,27]$ :

$$
X(m, n)=4.3 \times 10^{-6} f(n, m) \psi(n, m) \exp \left[-E(n, m) / T_{\mathrm{e}}\right] /\left[E(n, m) T_{\mathrm{e}}^{1 / 2}\right],
$$

the energy $E(n, m)=E(n)-E(m), m<n$ and $f(n, m)$ is the oscillator strength.

An effective Gaunt factor for ions follows the expression given by Blaha [28] and used elsewhere $[23,29]$ as

$$
\begin{aligned}
& Z_{0}=1-2 / z \\
& Z_{1}=1+Z_{0} \chi \\
& \psi(n, m)=0.19\left\{1+0.9\left[1+n(n-m) Z_{1} / 20\right] \exp \left(\chi_{m n}\right) E_{L}\left(\chi_{m n}\right)\right\}
\end{aligned}
$$

where $\chi_{m n}=E_{m n} / T$. The de-excitation rate is calculated from the detailed balance.

\subsection{Collisional ionization}

The collisional ionization from a specific level $m$ to a given level $n$ of the next ionization $(z)$ developed by Drawin [24] was used

$$
\begin{aligned}
& u=\frac{\Delta E}{T_{\mathrm{e}}}, \\
& S^{z-1}(m, n)=2.38 \times 10^{-8} E_{H}^{2} f(m) \xi(m) \psi(u, \beta) / \Delta E T_{\mathrm{e}}^{1 / 2},
\end{aligned}
$$

where $E_{H}=13.59 \mathrm{eV}, \xi(m)$ the number of equivalent electrons in the outer shell, $\Delta E=E_{z}(n)-E_{(z-1)}(m), f(m)$ the oscillator strength for an ionization from level $m$, and $\beta=1+[(z-1) /(z+1)]$. The function $\psi(u, \beta)$ has an integral form 
and it was approximated by the analytical expression [26] as

$$
\psi(u, \beta)=(1.0+u)^{-1}\left\{(20.0+u)^{-1}+\ln [1.25 \beta(1.0+1 / u)]\right\} .
$$

\subsection{Three-body recombination}

The rate coefficient for three-body recombination, obtained from the detailed balance, follows the expression given below

$$
\alpha_{3}^{z-1}(n, m)=1.66 \times 10^{-22} g^{z-1}(m) S^{z-1}(m, n) \exp (u) /\left[g^{z}(n) T_{\mathrm{e}}^{3 / 2}\right],
$$

where $g^{z}(k)$ is the statistical weight of state $k$ and ionization stage $z$.

\subsection{Radiative recombination}

The radiative recombination rate approximated by Bates et al. [22] was used in these calculations

$$
\alpha_{\mathrm{R}}^{z-1}(p, i)=5.2 \times 10^{-14} u^{3 / 2} \exp (u) E_{1}(u),
$$

where $E_{1}$ is the first exponential integral and expressed as

$$
E_{1}(u)=\int_{1}^{\infty} \mathrm{d} x \exp (u x) / x
$$

the approximated expression of $\exp (u) E_{1}(u)$ given by Itoh et al. [29] was used.

\subsection{Dielectronic recombination}

The rate of dielectronic recombination given by Post et al. [23] and Itoh et al. [29] was used in this study

$$
\gamma_{z, n}=2.40 \times 10^{-6} B\left(q_{z, n}\right) D\left(q_{z, n}, T\right) T_{\mathrm{e}}^{-3 / 2} \sum_{m} f_{m n} A(y) \exp \left(-E_{m n} / T_{\mathrm{e}}\right),
$$

where

$$
\begin{aligned}
& B(q)=q^{1 / 2}(q+1)^{5 / 2}\left(q^{2}+13.4\right)^{-1 / 2}, \quad E_{m n}=\left(E_{z, m}-E_{z, n}\right), \\
& a=1+0.015 q^{3} /(q+1)^{2}, \quad y=(q+1)\left(n^{-2}-m^{-2}\right), \\
& A(y)=0.5 y^{1 / 2} /\left(1+0.210 y+0.030 y^{2}\right), \\
& D\left(q, T_{\mathrm{e}}\right)=0.0015\left[(q+1) n_{t}\right]^{2} /\left\{1+0.0015\left[(q+1) n_{t}\right]^{2}\right\} \\
& n_{t}=1.508 \times 10^{17} q^{6} T_{\mathrm{e}}^{1 / 2} / N_{\mathrm{e}}
\end{aligned}
$$

\section{Lowering ionization potential}

The ionization energy of an isolated atom or ion is different from one immersed in a plasma [30] and must be corrected properly. These charged particles of plasma decrease the ionization level of isolated particles where some bounded levels appear in the continuum range. The lowering ionization potential is expressed as

$$
\Delta E_{\infty}^{(z-1)}=1.93 \times 10^{-10} z\left(N_{\mathrm{e}} / T_{\mathrm{e}}\right)^{1 / 2},
$$

where $z$ is the charge of the ion, $N_{\mathrm{e}}$ in $\mathrm{cm}^{-3}$ and $T_{\mathrm{e}}$ in $\mathrm{eV}$. 


\section{Opacity effect and gain}

For an exact calculation of opacity effect, the radiative transfer equation must be solved. In order to treat the equation of radiative transfer quantitatively, it is a common practice, as a first approximation, to introduce an escape factor $\Lambda_{n m}$ for each line, which is equal to 1 and 0 for optically thin and thick lines, respectively. This factor has been approximated by Holstein [31] and gives a good measure for lines with large optical thickness. However, for Doppler broadening, this formula [31] gives underestimated values (see Fig. 10 Ref. [32]) and it is not valid in the region of relatively small optical thickness. The formula given by Fujimoto [32], in the case of Doppler broadening and cylindrical geometry, was used and expressed as

$$
\Lambda_{p q}=\left\{1.92-1.3 /\left[1+\left(\tau_{p q}\right)^{6 / 5}\right]\right\}\left(\tau_{p q}+0.62\right)\left[\pi \ln \left(1.375+\tau_{p q}\right)\right]^{1 / 2},
$$

where $p$ and $q$ are the upper and lower lasing levels, respectively. $\tau_{p q}$ is the optical depth derived by Drawin et al. [26].

$$
\tau_{p q}=\pi^{1 / 2} e^{2} f_{p q} \lambda_{p q} n(p) L\left(M / 2 K T_{\mathrm{g}}\right)^{1 / 2} / m_{\mathrm{e}} c,
$$

where $f_{p q}$ is the oscillator strength, $m_{\mathrm{e}}$ and $M$ are the mass of electron and emitter particle, respectively, $T_{\mathrm{g}}$ is the temperature of particle and $L$ is the length of plasma. The relation of gain, in the case of Doppler profile and $T_{\mathrm{e}}=T_{\mathrm{g}}$ assumptions, is expressed for lasing transitions as

$$
G_{p q}=1 /(8 \pi c)\left(M c^{2} / 2 \pi K T_{\mathrm{g}}\right)^{1 / 2} g(p)[n(p) / g(p)-n(q) / g(q)] A_{p q} \lambda_{p q}^{3},
$$

where $\lambda_{p q}$ is the lasing wavelength between $p$ and $q$ levels.

\section{Results and discussion}

The total population of aluminum ions against electron temperature is shown in Fig. 2. At an electron density of $10^{18} \mathrm{~cm}^{-3}$ the total population of F-like increases while those from Na-like ions to aluminum atoms decrease when the electron temperature of plasma increases. However, the total population of Ne-like remains relatively constant over this wide range of temperature. Around the electron temperature of $10 \mathrm{eV}$, this plasma is dominated by Ne-like aluminum ions which agrees well with the results given by Duston et al. [33].

The calculated population density of levels for Ne-like Al ions, given in Fig. 3, shows the dominance of its ground state among other levels.

The relative population densities among excited states of $3 s$ and $3 p$ configurations, given in Fig. 4, show that the population inversion between $3 s^{u} P_{1}^{0}(u=3,1)$ and $3 p\left({ }^{3} P_{0},{ }^{1} S_{0}\right)$ levels can occur as predicted for other materials [3]. The population inversion rate reaches its highest value of 7.16 between transitions $3 s^{1} P_{1}^{0}$ and $3 p^{3} P_{0}$ levels at the electron density of $10^{19} \mathrm{~cm}^{-3}$.

The gain of lasing transitions, between $3 s{ }^{u} P_{1}^{0}(u=3,1)$ levels and $3 p\left({ }^{3} P_{0},{ }^{1} S_{0}\right)$ levels, has been calculated by using the analytical expression (17). Its evolution against plasma parameters without opacity effect, given in Fig. 5, shows that the gain magnitudes increase when the electron density and/or the electron temperature increase. 

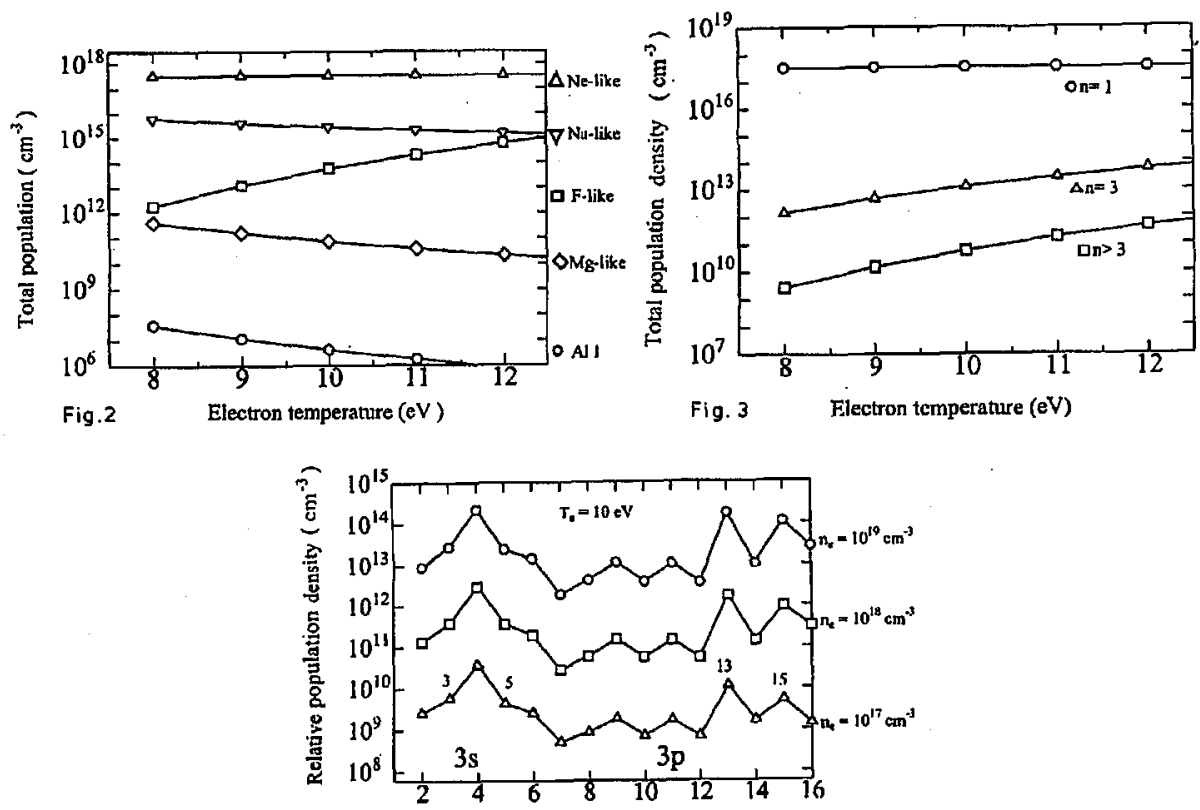

Fig. 4 Numbered levels of $3 \mathrm{~s}$ and $3 \mathrm{p}$ configurations

Fig. 2. Evolution of the total population of various ionization stages against electron temperature at $n_{\mathrm{e}}=10^{18} \mathrm{~cm}^{-3}$.

Fig. 3. Evolution of the total population density of ground and some excited states of Ne-like aluminum ions at $n_{\mathrm{e}}=10^{17} \mathrm{~cm}^{-3}$.

Fig. 4. Relative population densities of $3 s$ and $3 p$ levels at various electron density and $T_{\mathrm{e}}=10 \mathrm{eV}$. For their corresponding atomic configuration and numeration, refer to Fig. 1 and Table, respectively.

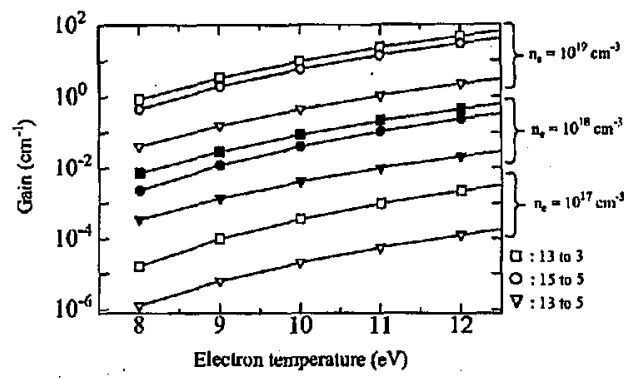

Fig. 5. Evolution of the gain against electron density and temperature without opacity effect. For their corresponding atomic configuration, refer to Fig. 1.

At a low electron density of $10^{17} \mathrm{~cm}^{-3}$. the gain between the upper level ${ }^{3} p^{3} P_{0}(13)$ and lower levels $3 s\left({ }^{3} P_{1}^{0},{ }^{1} P_{1}^{0}\right)(3,5)$ reaches a value smaller than $10^{-2} \mathrm{~cm}^{-1}$ at $T_{\mathrm{e}}=12 \mathrm{eV}$. However, at this electron density, the population in- 


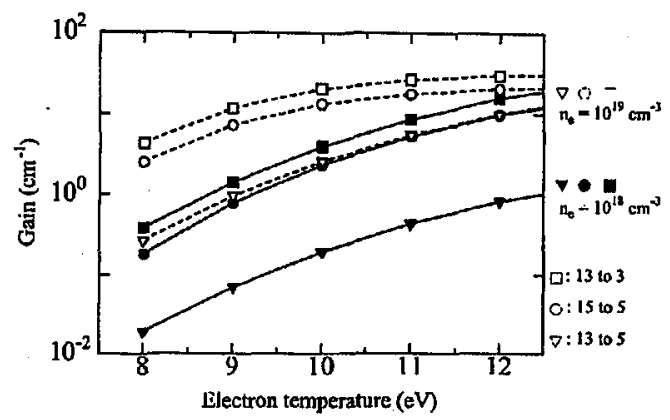

Fig. 6. Evolution of the gain against electron density and temperature with opacity effect. For their corresponding atomic configuration, refer to Fig. 1.

version between $3 p^{1} S_{0}(15)$ and $3 s^{1} P_{1}^{0}(5)$ levels has not occurred, because the values of electron collisional deexcitation rates from the levels $(17,23,27)$ (see Table) of $3 d$ configuration to the upper lasing level (15) were insufficient to establish the population inversion. Furthermore, the upper level (15) decays much faster to the lower level (5) than the transitions between upper level (13) and the lower levels $(3,5)$.

The deexcitation process from $3 d$ levels was among the dominant populating processes for the upper lasing levels of $3 p$ which has enhanced the gain magnitudes of Ne-like selenium lasing line $(J=2 \div 1)$ by $15-20$ percent [2].

At the high electron densities of $10^{18}$ and $10^{19} \mathrm{~cm}^{-3}$, the population densities of lasing levels (13) and (15) are enhanced by deexcitation process from $3 d$ levels as shown in Fig. 4. This enhancement improves the overpopulation between lasing levels $(13)$ to $(3,5)$ and strains the establishment of lasing between the levels (15) to (5). Consequently, at an electron density of $10^{19} \mathrm{~cm}^{-3}$ and $T_{\mathrm{emax}}=0.10 \times I_{0}$, where $I_{0}$ is the ionization limit of Ne-like ions, the gain values are greater than $1 \mathrm{~cm}^{-1}$ for both transitions (13) to (3) and (15) to (5). However, at the electron density of $10^{19} \mathrm{~cm}^{-3}$ and $T_{\mathrm{e}}=0.5 \times I_{0}$, the scaling law of gain for Ne-like system predicts a lower value smaller than $0.1 \mathrm{~cm}^{-1}$ (Fig. 3 of Ref. [4]).

The enhancement of gain values predicted by our CRE model is due to the pumping of upper levels $3 p$ by inner-shell ionization of Na-like [2] and by the dielectronic recombination from the ground state of $F$-like $[2,10,11]$. The used atomic structure for lasing ions, excited states up to $n=9$ and recently published atomic data for $n=3$, have a significant effect on the gain magnitudes.

The opacity effect on gain behavior, given in Fig. 6, shows that the gain values are slightly increased relatively to the optically thin values. It is due to an increase in the optical thickness of resonance lines from $3 d$ levels which has improved the gain values [34].

\section{Conclusion}

A VUV lasing on Ne-like of aluminum ions has been presented. The population inversion was established between $3 p$ and $3 s$ configurations. The highest gain has been found between $3 p^{0} P_{1}^{0}$ and $3 s^{3} P_{1}^{0}$ levels. The opacity effect alters dras- 
tically the gain values. The sensitivity of gain magnitudes on the atomic processes rates, collisional-radiative scheme and the line profile could not be omitted.

\section{Acknowledgment}

This work was supported by the Ministry of Education, Science, Culture and Sports of Japan. I wish to thank Tokyo Institute of Technology (T.I.T) and the University of Sciences and Technology of Oran (U.S.T.O/Algeria), which have allowed this collaboration. Thanks to Prof. S. Ishii for his invitation to the laboratory.

\section{References}

[1] A.V. Vinogradov, V.N. Shilyaptsev, Sov. J. Quantum Electron. 10, 754 (1980).

[2] A. Dasgupta, K.G. Whitney, M. Blaha, M. Buie, Phys. Rev A 46, 5973 (1992).

[3] U. Feldmann, A.K. Bhatia, S. Suckewer, J. Appl. Phys. 54, 2188 (1983).

[4] U. Feldmann, J.F. Seely, A.K. Bhatia, J. Appl. Phys. 56, 2475 (1984).

[5] J.P. Apruzese, J. Davis, Phys. Rev. A 28, 3686 (1983).

[6] G.D. Enrigth, D.M. Villeneuve, J. Dunn, H.A. Baldis J.C. Kieffer, H. Pepin, M. Chaker, P.R. Herman, J. Opt. Soc. Am. B 8, 2047 (1991).

[7] P.B. Holden, G.J. Pert, A.E. Kingston, E. Robertson, Appl. Phys. B 58, 23 (1994).

[8] J. Davis, R. Clark, J.P. Apruzese, P.C. Kepple, IEEE Tr. Plasma. Sci. 16, 482 (1988).

[9] J.W. Thornhill, J. Davis, J.P. Apruzese, R. Clark, Appl. Opt. 31, 4940 (1992).

[10] J.P. Apruzese, J. Davis, M. Blaha, P.C. Kepple, V.L. Jacobs, Phys. Rev. Lett. 55, 1877 (1985).

[11] G.P. Gupta, B.K. Sinha, J. Appl: Phys. 77, 2287 (1995).

[12] V. Kaufmann, W.C. Martin, J. Phys. Chem. Ref. Dat. 20, 809 (1991).

[13] A. Lindgard, S.E. Nielsen, At. Data Nucl. Data Tables 19, 533 (1977).

[14] A. Hibbert, M.Le. Dourneuf, M. Mohan, At. Data Nucl. Data Tables 53, 23 (1993).

[15] A.V. Loginov, P.F. Guzdev, Opt. Spectrosc. 45, 725 (1978).

[16] L.C. Johnson, Astrophys. J. 174, 227 (1972).

[17] L. LaJohn, Phys. Scr. 49, 169 (1994).

[18] B.C. Fawcett, Phys. Scr. 30, 326 (1984).

[19] M. Westerlend, N. Reistad, C. Jupen, L. Engstrom, Nucl. Instrum. Methods Phys. Res. B 31, 300 (1988).

[20] L. Vriens, A.H.M. Smeets, Phys. Rev. A 22, 3, 940 (1980).

[21] M.J. Seaton, Mon. Not. R. Astron. Soc. 119, 81 (1959).

[22] D.R. Bates, A.E. Kingston, R.W.P. McWhirter, Proc. R. Soc. Lond. A 267, 297 (1962).

[23] D.E. Post, R.V. Jensen, C.B. Tarter, W.H. Grasberger, W.A. Lokke, At. Data Nucl. Data Tables 20, 397 (1977).

[24] H.W. Drawin, Z. Phys. 225, 470 (1969).

[25] M. Gryzinski, Phys. Rev. 138, 336 (1965). 
[26] H.W. Drawin, F. Emard, Physica C 85, 333 (1977).

[27] R.D. Taylor, A.W. Ali, Appl. Phys. 64, 89 (1988).

[28] M. Blaha, Astrophys. J. 157, 473 (1969).

[29] M. Itoh, T. Yabe, Phys. Rev. A 35, 233 (1987).

[30] C. Pecker-Wimel, Introduction to the Plasma Spectroscopy, DUNOD, Paris 1967.

[31] T. Holstein, Phys. Rev. 83, 1159 (1951).

[32] T. Fujimoto, J. Quant. Spectrose; Radiat. Trans. 21, 439 (1978).

[33] D. Duston, J. Davis, Phys. Rev. A 21, 1664 (1980).

[34] A.V. Vinogradov, I.I. Sobelman, E.A. Yukov, Sov. J. Quantum Elektron. 7, 32 (1977). 